\title{
COMPARAÇÃO FITOSSOCIOLÓGICA ENTRE DUAS AMOSTRAGENS NUMA ÁREA DE CLAREIRA EM ANOS CONSECUTIVOS, ESTAÇÃO BIOLÓGICA DE CARATINGA, MG'
}

\author{
Leonardo Vianna da Costa e Silva2
}

Recebido em 19-09-91. Aceito em 13-10-93.

RESUMO - (Comparação fitossociológica entre duas amostragens numa área de clareira em anos consecutivos, Estação Biológica de Caratinga, MG). A Estação Biológica de Caratinga encontra-se dentro do domínio Atlântico, sob um clima sazonal com uma estação úmida e quente (outubro-março) e outra seca e fria (abril-setembro). O solo é do tipo Latossolo Vermelho-Amarelo álico. A clareira em estudo localiza-se em topo de morro, possuindo pequenas árvores esparsas, grande quantidade de "touceiras" mortas de Pteridium aquilinum, plantas herbáceas, jovens e plântulas de espécies arbóreas. Esta área foi alterada por fogo e plantio de café há pouco mais de 30 anos. Foram amostrados $500 \mathrm{~m}^{2}$ mapeando-se e anotando-se altura e circunferência de todos os indivíduos. Realizaram-se 2 amostragens com o mesmo método, a primeira em outubro de 1989 e a segunda em outubro de 1990 . Verificou-se pouca variação na densidade (657 e 668 indivíduos, respectivamente) e na composição em espécies. Mabea fistulifera (maior densidade em ambas as amostragens), Bauhinia fusco-nervis, Inga $s p$ e uma espécie não identificada de gramínea tiveram a densidade aumentada, enquanto Pteridium aquilinum (a segunda de maior densidade), Ferdinandusa $c f$. ruggeoides e Vismia $s p$, tiveram-na reduzida.

A presença de "touceiras" mortas de $P$. aquilinum, de indivíduos jovens de espécie arbóreas comuns às matas ao redor e a existência de áreas vizinhas ocupadas exclusivamente por populações de $P$. aquilinum sugerem que a clareira em estudo encontra-se em estágio intermediário entre o declínio da população de $P$. aquilinum e a ocupação da área pelas espécies de mata.

Palavras-chaves: clareira, sucessão, regeneração, floresta Atlântica, floresta semidecídua.

1 - Financiamento: Fundação Biodiversitas e Conservation International. Trabalho apresentado no XLII Congresso Nacional de Botânica.

2 - Depto. Botânica-UFMG, Av. Antonio Carlos, 6627, Cx. P. 2486, 31270-901 - Belo Horizonte, MG. 
ABSTRACT - (Phytosociology comparing between two gaps in the Caratinga Biologic Station, Minas Gerais State). The Estação Biológica de Caratinga is located in the Atlantic dominium, The climate is seasonal with a humid hot season (October-March) and a dry cold season (April-September). The soil is alic Yelowish-Red Latosol. The gap studied is located on the top of a hill, where there are small scattered trees, a large amount of dead bunches of Pteridium aquilinum, herbs, and saplings of tree species. The position of each individual was mapped, and its height and circunference were recorded in a plot of $500 \mathrm{~m} 2$. Two samples were made by the same method, the first in October 1989, and the second in October 1990. A small variation in density (657 to 668 individuals, respectively) as well as in the composition of the species was verified. Mabea fistulifera (the specie with the highest density in both samples), Bauhinia fusco-nervis, Mikania sp. and a non-identified species of Gramineae increased in density, while Pteridium aquilinum (the second highest density), Ferdinandusa cf. rudgeoides e Vismia sp. decreased in density. The presence of dead bunches of $P$. aquilinum, saplings of the species common to the sorrounding forests and the existence of $P$. aquilinum populations nearby all suggest that the gaps are in an intermediate stage between the decline of the population of $P$. aquilinum and the occupation of the area by the species od the forest.

Kew-words: gaps, regeneration, succession, Atlantic forest.

\section{Introdução}

A importância da regeneração de clareiras na estrutura, dinâmica e composição de florestas tropicais tem sido enfatizada por diversos autores. Estes, em sua maioria, enfocam o processo sob o ponto de vista qualitativo e em um tempo instantâneo (Browkaw, 1987), sem acompanhamento do processo de sucessão. Gómez-Pompa (1974 apud Castellani, 1986) enfatizou a importância de estudos que acompanhem o processo sucessional em parcelas permanentes.

Clements (1916 apud Kershaw \& Looney, 1985) acreditava que qualquer processo de sucessão dentro de um determinado ecossistema confluiria para um único clímax climático regional. Gleason (1926 apud Kershaw \& Looney, 1985) foi o primeiro a combater tal idéia, introduzindo o conceito individualista de associação de espécies, no qual o processo sucessional ocorreria em decorrência das variações apresentadas pelas populaçães de espécies ao longo do tempo e do espaço.

Diversos fatores influem na determinação de quais espécies colonizarão com sucesso determinada clareira: tamanho da clareira; mecanismo de germinação das sementes; período de formação da clareira relacionado ao periodo de frutificação das espécies; proximidade à fonte de sementes e condições de nutrientes do solo (Whitmore, 1982, Pickett, 1983, apud Castellani, 1986).

Em áreas de floresta tropical, dependendo do grau e do tipo de ação pertubatória pode ocorrer a sucessão desviada, caracterizada por uma comunidade final dominada por ervas e arbustos resistentes ao fogo (Budowski, 1966). 
Dentro da situação de devastação em que se encontra a Floresta Atlântica (Fonseca, 1985), é necessário conhecer o processo de sucessão natural, criando parâmetros para efetuar a recuperação de áreas potenciais.

Este estudo propõe avaliar a variação estrutural da vegetação em uma clareira através da comparação de alguns parâmetros fitossociológicos de duas amostragens em anos consecutivos na mesma área, criando subsídios para melhor compreensão do processo de regeneração natural em florestas.

\section{Material e métodos}

Área de estudo - A Estação Biológica de Caratinga (EBC) localiza-se na Fazenda Montes Claros, município de Caratinga, leste de Minas Gerais, a $19^{\circ} 50^{\prime} \mathrm{S}$ e $41^{\circ} 50^{\prime} \mathrm{W}$, dentro do domínio Atlântico. É uma área de cerca de 880 ha, separada de várias outras pequenas manchas florestais por pastagens e/ou lavouras, ou por áreas em estádios iniciais de sucessão. A topografia é montanhosa com altitudes variando entre 318 e $682 \mathrm{~m} \mathrm{e}$ variada composição florística (Silva, 1988).

O clima é tipicamente sazonal, apresentando um período seco com temperaturas médias mensais de 15 a $20^{\circ} \mathrm{C}$ (abril-setembro) e um período chuvoso de temperaturas médias mensais entre 20 e $22^{\circ} \mathrm{C}$ (outubro-março). A precipitação média anual encontrase por volta de $1146,3 \mathrm{~mm}$ (Ferrari, 1988). O solo é Latossolo Vermelho-Amarelo álico (RADAMBRASIL, 1982).

A vegetação da $E B C$ é composta por matas primárias e áreas secundárias em diferentes estádios de sucessão, que variam desde clareiras quase completamente ocupadas por Pteridium aquilinum até matas de grande porte, com formações semidecíduas nas maiores altitudes e perenifólias nos fundos de vales. Segundo moradores da região, algumas áreas hoje cobertas por florestas foram cafezais há mais de 40 anos atrás e parte da mata sofreu um último grande incêncio há cerca de 30-40 anos. Estes fatos, acrescidos ao corte seletivo de árvores, são os fatores responsáveis pela presenção de vegetação secundária na EBC.

A área amostrada localiza-se em alto de morro, em encosta voltada para norte, com presença de plantas herbáceas, arbustivas, indivíduos jovens de espécies arbóreas e grande quantidade de "touceiras" mortas de $P$. aquilinum. Esta área, além de ter sido cultivada com cafezais, também foi atingida pelo incêndio. A área caracteriza-se como clareira, que é definida por Brokaw (1982) como uma abertura vertical na floresta estendendo-se através dos níveis de folhagem até $2 \mathrm{~cm}$ do solo.

Foram demarcados 20 quadrados contíguos de $5 \times 5 \mathrm{~m}\left(25 \mathrm{~m}^{2} / \mathrm{cada}\right)$, totalizando uma área de $500 \mathrm{~m}^{2} \mathrm{em}$ uma clareira de cerca de $550 \mathrm{~m}^{2}$. Todos os indivíduos presentes, desde plântulas até árvores adultas, foram amostrados, mapeados e medida sua altura. As "touceiras" de gramíneas e ciperáceas foram consideradas como um indivíduo. A primeira amostragem (A1) foi realizada em outubro/1989 e a segunda (A2), utilizando o mesmo método, em outubro/1990. Os individuos presentes em A1 e não encontrados em A2 foram considerados como mortos e os amostrados em A2 e ausentes em Al, representaram os novos recrutas. A espécie $P$. aquilinum recebeu tratamento diferenciado devido à dificuldade de distinguir seus indivíduos, pois a reprodução vegetativa através de rizomas subterrâneos é freqüente nesta espécie, formando extensas colônias (Fletcher \& Kirkwood, 
1979). Cada folha desta espécie foi tomada como um individuo, não sendo medida sua altura e não considerando os indivíduos mortos e recrutas.

Foram calculados a densidade total e por espécie e 0 índice de diversidade de Shannon através da fórmula $\mathrm{H}^{\prime}=-\Sigma(\mathrm{ni} / \mathrm{N}) \cdot(\ln \mathrm{ni} / \mathrm{n})$, onde ni é o número de indivíduos amostrados da espécie i, $\mathrm{N}$ é o número total de indivíduos amostrados e ln é o logarítimo neperiano (Cavassan et al., 1984). A significância da variação no índice de diversidade foi testada através do teste $t$ (Zar, 1984).

A distribuição em classes de altura é apresentada em figura gráfica, sendo a amplitude do intervalo definida através da fórmula de Yule $(2,5 \times \sqrt[4]{\mathrm{N}})$, onde $\mathrm{N}$ é o número de amostras.

\section{Resultados e discussão}

A extensão da clareira $(550 \mathrm{~m} 2)$ favorece uma intensa penetração de luz em seu interior. Nestas condições, espera-se que espécies pioneiras, que necessitam de luz para germinar sejam favorecidas, em detrimento daquelas que apenas germinam à sombra.

As espécies encontradas na clareira foram consideradas como pioneiras ou intolerantes à sombra segundo critério de Whitmore (1989) e variaram desde espécies herbáceas/arbustivas de curto ciclo de vida, como é o caso de Mikania sp., Leandra $\mathrm{cf}$. erinacea, e algumas gramíneas e ciperáceas, até i ndivíduos de espécies arbóreas de longo período de vida, como Mabea fistulifera e Anadenanthera colubrina. Estas últimas poderiam ser denominadas secundárias tardias, conforme as características definidas por Budowski (1965), sendo ambas de grande ocorrência nas matas de topo de morro da Estação.

Foi amostrado um total de 60 espécies (Tabela 1), sendo que 4 delas apenas ocorreram em A1 e outras 4, apenas em A2. Apesar deste número, a predominância de Mabea fistulifera e Pteridium aquilinum resultou em uma pequena diversidade $(\mathrm{H}=2,55$ nats/indivíduo em $\mathrm{A} 1 \mathrm{e} \mathrm{H}=2,50$ nats/indivíduo $\mathrm{em} \mathrm{A2}$ ). Através do teste t esta variação entre as duas amostras mostrou-se não significativa, $\mathrm{t}=1,295 \mathrm{e} \mathrm{gl}=1234,99$.

A baixa densidade de indivíduos encontrada em ambas as amostragens (657 e 668 indivíduos, Tabela 1), o reduzido tamanho da copa (principalmente em M. fistulifera) e 0 pequeno porte da maioria dos indivíduos (Figura 1) permitiriam uma alta incidência luminosa sobre o solo, favorecendo desta forma a germinação de espécies pioneiras.

A pouca variação apresentada pelos parâmetros analisados mostra que a resultante do processo de regeneração nesta área durante o intervalo de um ano foi quase nula, mascarando, de certa forma, o processo dinâmico de morte e recrutamento existente nos indivíduos das espécies amostradas (Tabela 1$)$. $O$ número de recrutas (112, representando $16,8 \%$ dos indivíduos amostrados em A2) e o de mortos (93, 14,2\%) encontrados no intervalo de um ano, apesar de demostrarem estar havendo germinação e morte na área, não indicam necessariamente a dinâmica neste periodo. Provavelmente, neste intervalo ocorreram plantas que germinaram, mas devido a alguma pressão ambiental não se estabeleceram. 


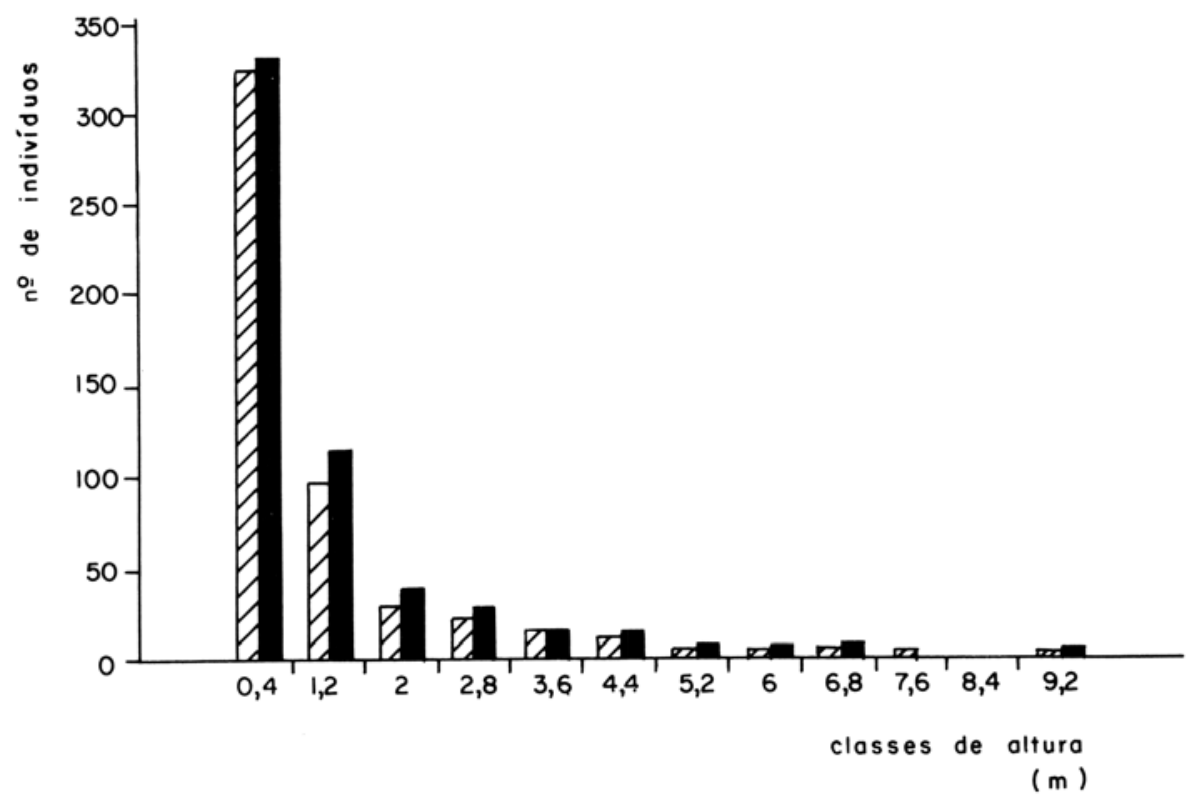

Figura 1 - Distribuição em classes de altura dos indivíduos presentes na clareira, Estação Biológica de Caratinga, MG, em duas amostragens consecutivas:

A1 $-1^{\text {a }}$ amostra (out./1989)

A2 - 2 amostra (out./1990)

Ó́ndice relativo de mortalidade encontrado na clareira em estudo, quando comparado com dados da literatura, apresenta-se bastante baixo. Ausgspurger (1983 apud Runkle, 1989) encontrou entre 88 e $94 \%$ de mortalidade; Clark \& Clark (1984, apud Runkle, 1989), $90 \%$ e Connel et al. (1984, apud Runkle, 1989) entre 20 e $50 \%$. Este fato poderia estar relacionado com a época da amostragem, pouco antes da estação chuvosa, quando muitos dos indivíduos que poderiam ter germinado no período anterior talvez não tivessem suportado um estresse hídrico durante a estação seca, não sendo, portanto, cmputados. 
Tabela 1 - Espécies presentes na clareira e respectivos parâmetros em duas amostragens (A1 em 1989 e A2 em 1990). Estação Biológica de Caratinga, MG. arb=arbusto, av=árvore, NI=não identificada

\begin{tabular}{|c|c|c|c|c|c|c|c|}
\hline \multirow{2}{*}{ Espécie } & \multirow[t]{2}{*}{ Hábito } & \multicolumn{2}{|c|}{$\begin{array}{c}\mathrm{N}^{\circ} \mathrm{de} \\
\text { indivíduos }\end{array}$} & \multicolumn{2}{|c|}{$\begin{array}{l}\text { Altura média } \\
(\mathrm{m})\end{array}$} & \multicolumn{2}{|c|}{ Dinâmica } \\
\hline & & $\mathrm{Al}$ & $\mathrm{A} 2$ & $\mathrm{~A} 1$ & $\mathrm{~A} 2$ & morte & $\begin{array}{l}\text { recru- } \\
\text { tas }\end{array}$ \\
\hline Mabea fistulifera Mart. & av & 231 & 247 & 1.07 & 1.12 & 20 & 36 \\
\hline Pteridium aquilinum (L.) Kuhn & erva & 133 & 125 & 110 & 1.09 & - & - \\
\hline Bauhinia fusco-nervis (Bong.) Stend. & av & 29 & 36 & 1.01 & 0.09 & 4 & 11 \\
\hline Ferdinandusa rudgeoides (Benth.) Weec & ed av & 37 & 30 & 1.17 & 1.42 & 8 & 1 \\
\hline Mikania sp. 1 & liana & 14 & 20 & - & - & 6 & 12 \\
\hline Leandra cf. erinacea Cogn. & arb & 25 & 18 & 1.18 & 1.15 & 9 & 2 \\
\hline Cyperaceae 1 & erva & 16 & 16 & 0.34 & 0.51 & 3 & 3 \\
\hline Gramineae 3 & erva & 4 & 15 & 0.12 & 0.22 & 0 & 11 \\
\hline Guatteria sp. 1 & av & 14 & 14 & 1.92 & 1.71 & 2 & 2 \\
\hline Machaerium brasiliense Vog. & av & 12 & 13 & 0.52 & 0.61 & 2 & 3 \\
\hline Vismia sp. & av & 15 & 12 & 0.65 & 0.65 & 6 & 3 \\
\hline Xylopia sericea St. Hill & av & 9 & 11 & 0.87 & 0.97 & 1 & 3 \\
\hline Sclerolobium rugosum Mart. ex. Benth & av & 6 & 8 & 0.38 & 0.35 & 2 & 4 \\
\hline Dyctioloma incanescens $\mathrm{DC}$ & av & 7 & 8 & 2.13 & 2.18 & 0 & 1 \\
\hline Leandra sp. 1 & arb & 17 & 6 & 0.51 & 0.49 & 11 & 0 \\
\hline Davilla sp. & liana & 6 & 6 & - & - & 1 & 1 \\
\hline Erythroxylum spp. & av & 7 & 6 & 0.67 & 0.70 & 1 & 0 \\
\hline Maprounea guyanensis Aubl. & av & 3 & 5 & 1.37 & 1.03 & 0 & 2 \\
\hline Piptocarpha tomentosa Baker & av & 4 & 5 & 2.30 & 2.62 & 0 & 1 \\
\hline Graminae 1 & erva & 4 & 5 & 0.13 & 0.26 & 2 & 3 \\
\hline Cordia sellowiana Cham. & av & 8 & 5 & 0.74 & 1.08 & 3 & 0 \\
\hline Psychotria verticillata Muell. Arg. & arb & 4 & 4 & 0.58 & 1.12 & 0 & 0 \\
\hline Smilax sp. & liana & 0 & 4 & • & - & 0 & 4 \\
\hline Compositae 2 & arb & 5 & 4 & - & - & 1 & 0 \\
\hline Graminae 2 & erva & 4 & 4 & 0.55 & 1.02 & 0 & 0 \\
\hline Miconia subvernicosa $\operatorname{Cogn}$. & av & 3 & 3 & 1.47 & 1.53 & 0 & 0 \\
\hline Stryphnodendron polyphyllum Mart. & av & 3 & 3 & 0.10 & 0.11 & 2 & 2 \\
\hline Bignoniaceae 1 & liana & 2 & 3 & • & - & 0 & 1 \\
\hline Pogonophora schomburkiana Miers & av & 2 & 2 & 1.35 & 1.50 & 0 & 0 \\
\hline Byrsonima sp. & av & 2 & 2 & 1.03 & 1.85 & 0 & 0 \\
\hline Myrcia sp. 1 & av & 2 & 2 & 0.45 & 1.03 & 0 & 0 \\
\hline Myrcia rufula $\mathrm{Miq}$ & av & 2 & 2 & 1.95 & 2.32 & 0 & 0 \\
\hline Serjania cf. acutidentata Radlk. & liana & 1 & 2 & • & - & 0 & 1 \\
\hline NI 8 & $?$ & 1 & 1 & 0.10 & 0.15 & 0 & 0 \\
\hline NI 7 & $?$ & 0 & 1 & 0.10 & 0 & - & 1 \\
\hline
\end{tabular}


Cont. Tabela 1

\begin{tabular}{|c|c|c|c|c|c|c|c|}
\hline \multirow{2}{*}{ Espécie } & \multirow[t]{2}{*}{ Hábito } & \multicolumn{2}{|c|}{$\begin{array}{c}\mathrm{N}^{\circ} \text { de } \\
\text { individuos }\end{array}$} & \multicolumn{2}{|c|}{$\begin{array}{l}\text { Altura média } \\
\text { (m) }\end{array}$} & \multicolumn{2}{|c|}{ Dinâmica } \\
\hline & & $\mathrm{A} 1$ & $\mathrm{~A} 2$ & $\mathrm{~A} 1$ & $\mathrm{~A} 2$ & morte & $\begin{array}{c}\text { recru- } \\
\text { tas }\end{array}$ \\
\hline Leguminosae 2 & av? & 1 & 1 & 1.20 & 1.40 & 0 & 0 \\
\hline NI 2 & av & 1 & 1 & 0.40 & 1.60 & 0 & 0 \\
\hline Kielmeyera cf. petiolares Mart. & av & 1 & 1 & 1.30 & 1.60 & 0 & 0 \\
\hline Inga captata Desv & av & 2 & 1 & 0.15 & 0.10 & 2 & 1 \\
\hline Anadenanthera colubrina (Vell.) Bernan & $\mathrm{m}$ av & 1 & 1 & 8.50 & 9.00 & 0 & 0 \\
\hline Myrtaceae 1 & av & 1 & 1 & 0.50 & 0.95 & 0 & 0 \\
\hline Swartzia flaemingui Raddi & av & 1 & 1 & 4.00 & 4.30 & 0 & 0 \\
\hline Sapotaceae 2 & av & 1 & 1 & 2.00 & 3.80 & 0 & 0 \\
\hline Vitex sp. & av & 1 & 1 & 4.50 & 3.90 & 0 & 0 \\
\hline Compositae 1 & arb & 2 & 1 & 1.55 & 1.30 & 1 & 0 \\
\hline Cyperaceae 2 & erva & 0 & 1 & - & 0.20 & 0 & 1 \\
\hline Leguminosae 1 & av? & 0 & 1 & - & 0.70 & 0 & 1 \\
\hline Ouratea polygina Engler & av & 1 & 1 & 1.00 & 0.70 & 0 & 0 \\
\hline Marlierea tomentosa Camb. & av & 1 & 1 & 1.00 & 1.00 & 0 & 0 \\
\hline Rheedia gardneriana $\operatorname{Tr} \&$ PI & av & 1 & 1 & 0.60 & 0.90 & 0 & 0 \\
\hline Aparisthimium cordatum (A.Juss) Baill. & av & 1 & 1 & 0.20 & 0.50 & 0 & 0 \\
\hline NI 1 & $?$ & 0 & 1 & - & 0.15 & 0 & 1 \\
\hline Jacaranda puberula Cham & av & 1 & 1 & 1.70 & 2.00 & 0 & 0 \\
\hline Mollinedia $\mathrm{cf}$. oligantha Perking & av & 1 & 1 & 0.90 & 1.20 & 0 & 0 \\
\hline Casearia arborea (LC Richard) Urban & av & 1 & 1 & 0.70 & 0.20 & 0 & 0 \\
\hline NI 5 & $?$ & 1 & 0 & 0.20 & - & 1 & 0 \\
\hline Sapindaceae 2 & liana & 1 & 0 & 0.60 & - & 1 & 0 \\
\hline $\mathrm{NI} 4$ & $?$ & 1 & 0 & 0.05 & - & 1 & 0 \\
\hline NI 6 & $?$ & 1 & 0 & 0.40 & - & 1 & 0 \\
\hline NI 3 & $?$ & 2 & 0 & 0.40 & - & 2 & 0 \\
\hline Número de indivíduos & & 657 & 668 & & & & \\
\hline Diversidade - H’(Nat/indivíduo) & & 2.55 & 2.50 & & & & \\
\hline Recrutas & & & & & & & 112 \\
\hline Mortos & & & & & & 93 & \\
\hline
\end{tabular}

Apesar de os dados não apontarem uma significativa redução na população de Pteridium aquilinum (baseado em seu número de folhas) a presença na clareira de "touceiras" mortas e a ocorrência de áreas próximas totalmente ocupadas por esta espécie podem sugerir que a dinâmica de regeneração esteja passando por um final de ciclo na população de $P$. aquilinum e iniciando outra fase através dos indivíduos de espécies 
arbóreas. Dentre estas últimas destaca-se a predominância de Mabea fistulifera, a qual é citada por Silva (1988) como a espécie de maior IVI nas matas de topo de morro da Estação. Watt (1976, apud Fletcher \& Kirkwood, 1979) sugere que, quando a produção de serrapilheira em populações de $P$. aquilinum supera sua decomposição, esta espécie pode ser levada à degeneração, sendo substituída por outras. Dentro deste contexto, a clareira poderia estar em situação intermediária entre uma fase ocupada pela população de $P$. aquilinum e a das matas existentes na $\mathrm{EBC}$, onde $M$. fistulifera predomina.

A presença de poucos indivíduos nas maiores classes de altura (Figura 1) sugere que o processo de estabelecimento de espécies arbóreas nesta clareira vem acontecendo já há algum tempo, mas de forma isolada e ocasional. Possivelmente ao longo da existência desta clareira ocorreram alguns poucos "sítios seguros" (safe site), que preencheram as condições ambientais requeridas para a germinação das sementes e estabelecimento de alguns indivíduos (Harper, 1983). A título de comparação, para exemplificar a baixa velocidade deste processo na área estudada, considerando-se a interferência por fogo há cerca de 30 anos, cita-se o trabalho de Castellani (1986) em floresta semidecídua de Campinas (SP), onde em apenas 2 anos após o fogo a autora já indica árvores de até $6 \mathrm{~m}$ de altura e alto índice de cobertura.

Apesar de ocorrer de forma dinâmica, o processo de reocupação pela floresta na área em estudo acontece de forma lenta. Há cerca de 30 anos houve um incêndio, não havendo posteriormente nenhum tipo de pertubação. Desde então desenvolve-se a regeneração a até hoje neste local a vegetação não se encontra com porte florestal, sendo que no seu entorno a mata, que também foi área de plantio de café e sofreu incêndio, encontra-se com um dossel de até $20 \mathrm{~m}$ de altura. Isto leva a considerar que a população de $P$. aquilinum, colonizando a área em estudo, vem retardando a formação florestal, mantendo a sucessão em uma etapa de pioneiras iniciais por um período mais longo.

Como colocam Huston \& Smith (1987), a sucessão é autogênica, ou seja, a colonização por certas espécies em determinado ambiente modifica as características deste ambiente, fazendo com que outras espécies sejam inibidas ou favorecidas pela nova situação. No caso da área em estudo, a colonização por $P$. aquilinum poderia inibir o estabelecimento de outras espécies e, com o declínio desta população, haveria a possibilidade do surgimento das demais. Porém, esta hipótese carece de maiores estudos para o esclarecimento do mecanismo de ação e suas variáveis.

\section{Agradecimentos:}

Àna Elisa Brina, Ludmila M. S. Aguiar e Carlos Victor Mandonça Filho, pela ajuda de campo; ao José e Adriana Rimoli, Eduardo e Simone Veado pelo acolhimento; a Pedro Ivo Soares Braga pelo incentivo,a Fernando Roberto Martins e assessores da Revista, pelas pertinentes críticas e sugestões e, à Ruth Maria Pires Altoé pelo apoio de campo e na vida. 


\section{Referências bibliográficas}

Brokan, N.V. L. 1982. The definition of tree gap and its effect on measures of forest dynamics. Biotropica 14(2):158-160.

Brokan, N.V.L. 1987. Gap-phase regeneration of three pionner tree species in a tropical forest. Journal of Ecology 75:9-19.

Budowski, G. 1965. Distribuition of tropical American rain forest species in the.light of succesional process. Turrialba 15(1): 40-42.

Budowski, G. 1966. Los bosques de los tropicos húmedos de América. Turrialba 16:278285.

Castellani, T.T. 1986. Sucessão secundária inicial em mata tropical semi-decídua, após pertubação por fogo. Tese de Mestrado. Universidade Estadual de Campinas, Campinas.

Cavassan, O., Cesar, O. e Martins, F.R. 1984. Fitossociologia da vegetação arbórea da Reserva Estadual de Bauru, estado de São Paulo. Revta Brasil. Bot. 7(2):91-106.

Ferrari, S.F. 1988. The behavior and ecology of the buffy-headed marmoset Callitrix flaviceps (O. Thomas, 1903). Tese de Doutorado. Dept. of Anthropology University College, London.

Fletcher, W. W. \& Kirkwood, R.C> 1979. The braken fern (Pteridium aquilinum L. [Kuhn]); its biology and control. In: Dyer, A.F. The experimental biology of ferns. Academic Press. New York.

Fonseca, G.A.B. 1985. The vanishing Brazilian Atlantic forest. Biological Conservation 34:17-34.

Harper, J.L. 1983. Population Biology of plants. Academic Press, New York. P. 111113.

Huston, M. \& Smith, T. 1987. Plant sucession: life history and competition. American Naturalist 130(2): 168-198.

Kershaw, K.A. \& Looney, J.H. 1985. Quantitative and dynamic plant ecology. 3rd ed. Edward Arnold, Caulfield. 282 pp.

Radambrasil. 1982. Levantamento de recursos naturais. Mapa exploratório de solos. V. 34. Folha SE-24 Ed. Gráfica Barbero, Rio de Janeiro.

Runkle, J.R. 1989. Syncrony of regeneration, gaps, and latidudinal differences in tree especies diversity. Ecology 70(3): 546-547.

Silva, L.V.C. 1988. Variação fitossociológica de matas em gradiente altitudinal, Estação Biologica de Caratinga, MG. Monografia de Bacharelado. Universidade Federal de Minas Gerais, Bel Horizonte.

Whitmore, T.C. 1989. Canopy gaps and the two major groups of forest trees. Ecology 70(3):536-538

Zar, J. 1984. Biostatiscal Analysis. 2nd ed. Prentice-hall, Enlewood cliffs. P. 146-148. 